\title{
Immunotherapy with Aducanumab Restores Calcium Homeostasis in Tg2576 Mice
}

\author{
Ksenia V. Kastanenka, ${ }^{1}$ Thierry Bussiere, ${ }^{2}$ Naomi Shakerdge, ${ }^{1}$ Fang Qian, ${ }^{2}$ Paul H. Weinreb, ${ }^{2}$ Ken Rhodes, ${ }^{2}$ \\ and Brian J. Bacskai ${ }^{1}$ \\ ${ }^{1}$ Department of Neurology, MassGeneral Institute of Neurodegenerative Diseases, Massachusetts General Hospital and Harvard Medical School, \\ Massachusetts 02129, and ${ }^{2}$ Biogen, Cambridge, Massachusetts 02142
}

Calcium homeostasis plays a major role in maintaining neuronal function under physiological conditions. Amyloid- $\beta$ (A $\beta$ ) initiates pathological processes that include disruption in intracellular calcium levels, so amelioration of the calcium alteration could serve as an indirect functional indicator of treatment efficacy. Therefore, calcium dynamics were used as a measure of functional outcome. We evaluated the effects of the anti-A $\beta$ antibody aducanumab on calcium homeostasis and plaque clearance in aged Tg2576 mice with in vivo multiphoton imaging. Acute topical application of aducanumab to the brain resulted in clearance of amyloid plaques. Although chronic systemic administration of aducanumab in 22-month-old mice did not clear existing plaques, calcium overload was ameliorated over time. Therefore, this antibody likely restores neuronal network function that possibly underlies cognitive deficits, indicating promise as a clinical treatment. In addition, functional readouts such as calcium overload may be a more useful outcome measure to monitor treatment efficacy in models of Alzheimer's disease compared with amyloid burden alone.

Key words: aducanumab; Alzheimer's disease; amyloid; BIIB037; calcium

\section{Significance Statement}

Alzheimer's disease $(\mathrm{AD})$ is a progressive neurodegenerative disorder that is currently without a cure. Aducanumab is an antiamyloid- $\beta$ antibody being developed for the treatment of $\mathrm{AD}$. Interim analyses of a phase $1 \mathrm{~b}$ clinical trial have suggested potential beneficial effects on amyloid pathology and cognitive status in patients treated with aducanumab (Sevigny et al., 2016). Here, we show that a murine analog of aducanumab clears amyloid plaques in an acute setting and restores calcium homeostasis disrupted in a mouse model of $\mathrm{AD}$ upon chronic treatment. Therefore, we demonstrate that aducanumab reverses a functional outcome measure reflective of neural network activity.

\section{Introduction}

Alzheimer's disease (AD) is a progressive neurodegenerative disorder characterized by the presence of amyloid plaques and neurofibrillary tangles. Transgenic mouse models overexpressing amyloid precursor protein (APP) and developing amyloidrelated pathologies over time are valuable systems to investigate amyloid-targeted approaches for the treatment of AD. Anti-

Received June 29, 2016; revised Oct. 21, 2016; accepted 0ct. 27, 2016.

Author contributions: K.V.K., T.B.,P.H.W., and B.J.B. designed research; K.V.K. and N.S. performed research; T.B.,

F.Q., P.H.W., K.R., and B.J.B. contributed unpublished reagents/analytic tools; K.V.K. and N.S. analyzed data; K.V.K.,

T.B., P.H.W., and B.J.B. wrote the paper.

This work was supported by Biogen, the Alzheimer's Association, and the National Institutes of Health (Grants R01EB000768, R01AG044263, P30NS045776, and S10RR025645).

This research was funded by Biogen. The authors declare no competing financial interests.

Correspondence should be addressed to either Brian J. Bacskai or Ksenia V. Kastanenka, Department of Neurology, MassGeneral Institute of Neurodegenerative Diseases, Massachusetts General Hospital and Harvard Medical School, 114 Sixteenth St., Charlestown, MA 02129. E-mail: bbacskai@mgh.harvard.edu or kkastanenka@mgh.harvard.edu.

DOI:10.1523/JNEUROSCI.2080-16.2016

Copyright $\odot 2016$ the authors $\quad 0270-6474 / 16 / 3612549-10 \$ 15.00 / 0$ amyloid- $\beta(\mathrm{A} \beta)$ immunotherapy has shown the ability to prevent the formation of pathology in mouse models (Bard et al., 2000, Dodart et al., 2002, Bard et al., 2003, Wisniewski and Goni, 2014) and there is tremendous promise in this approach to prevent, if not reverse, the progression of AD in humans. Amyloid plaque clearance has been one of the primary end points of therapeutic efficacy in most preclinical studies but often has not translated into therapeutic efficacy in clinical trials (Doody et al., 2014, Salloway et al., 2014, Wisniewski and Goni, 2014). This disconnect emphasizes the need to introduce alternative and relevant functional end points during the preclinical evaluation of therapeutic candidates. We have previously reported that $\sim 20 \%$ of neurites (neuronal dendrites and axons) exhibit elevated levels of baseline calcium (calcium overload) that is correlated with disrupted neuronal structure and function in aged APP mice, a model that develops an amyloid pathology similar to that seen in AD (Kuchibhotla et al., 2008). Because increased calcium levels relate to neuronal activity, amelioration of this calcium overload could serve as an indirect functional indicator of treatment effi- 
cacy. To this end, we evaluated the effects of ${ }^{\text {ch }}$ aducanumab, a murine analog of the anti-A $\beta$ antibody aducanumab, on calcium homeostasis and plaque clearance in Tg2576 mice (Dunstan et al., 2011).

Aducanumab is a human antibody that binds selectively to aggregated forms of $\mathrm{A} \beta$, including insoluble fibrils and soluble oligomers. In a small phase $1 \mathrm{~b}$ study in prodromal or mild AD subjects, aducanumab was shown to reduce amyloid deposition in the brain in a dose- and time-dependent manner and to slow cognitive decline in a dose-dependent manner (Sevigny et al., 2016). chaducanumab, the murine chimeric analog of aducanumab, was used in the present rodent study because it accurately mimics immune effector function in mice. ${ }^{\text {ch }}$ aducanumab was applied directly onto the brain of living 22-month-old transgenic Tg2576 mice. Multiphoton microscopy (Kastanenka et al., 2015) showed clearance of existing amyloid plaques 3 weeks after

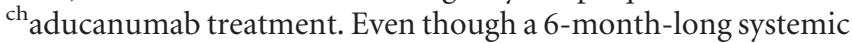
treatment with chaducanumab did not lead to amyloid plaque clearance, calcium overload in neurons in Tg2576 mice was ameliorated. This suggests that ${ }^{\mathrm{ch}}$ aducanumab may be targeting soluble species of $\mathrm{A} \beta$ and slowing cognitive decline by restoring calcium homeostasis and proper neuronal function.

\section{Materials and Methods}

Animals and surgery. Transgenic Tg2576 mice (APPSwe) at 18 or 22 months of age and nontransgenic control mice at 13 months of age were used (Taconic, RRID:IMSR_TAC:1349). Both sexes were included in the studies. The studies were conducted in accordance with Massachusetts General Hospital Animal Care and Use Committee and National Institutes of Health guidelines for the use of experimental animals.

In the acute immunotherapy condition, animals underwent a craniotomy and durotomy and received a topical application of the antibody as in previous experiments (Bacskai et al., 2001). IgG2a murine chimeric

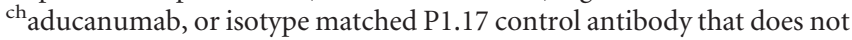
recognize an epitope in mouse were applied to the brains. Then, animals underwent cranial window implantation over somatosensory cortex as described previously (Kuchibhotla et al., 2008). Briefly, mice were anesthetized with isoflurane and placed in a stereotaxic apparatus. Their body temperature was maintained with a heating pad throughout the course of anesthesia. The scalp was disinfected with betadine and isopropyl alcohol before incision. A round window was made over the brain by drilling and removing the skull. Each mouse received a topical application of the antibody $(0.42-1 \mathrm{mg} / \mathrm{ml})$ to the brain for $20 \mathrm{~min}$. Subsequently, the site was covered with a glass coverslip $8 \mathrm{~mm}$ in diameter and fixed with a mixture of dental cement and crazy glue. One day before the craniotomy, animals received an intraperitoneal injection of methoxy-XO4 (10 mg/ $\mathrm{kg}$ ), which crossed the blood-brain barrier and labeled amyloid plaques (Klunk et al., 2002). Mice were 22 months old when they received the acute antibody treatment.

In the chronic immunotherapy condition, animals received intraperitoneal injections of the antibody (described below) after intracortical virus injections and cranial window installation as described previously (Kuchibhotla et al., 2008). Briefly, before AAV8-CBAYC3.6 (University of Pennsylvania) injections, mice were anesthetized with isoflurane and secured in a stereotaxic apparatus. The scalp was disinfected over the injection site. Burr holes were drilled through the skull in each hemisphere with the following coordinates A-P 0, $\mathrm{M}-\mathrm{L} \pm 1, \mathrm{D}-\mathrm{V}-1.5$ with respect to bregma. Using a Hamilton syringe, $1-1.5 \mu \mathrm{l}$ of virus $\left(2 \times 10^{12}\right.$ molecules $\left./ \mathrm{ml}\right)$ was injected $1.5 \mathrm{~mm}$ below the surface of dura at a rate of $0.13 \mu \mathrm{l} / \mathrm{min}$ to target neurons in the somatosensory cortex. After injections, a cranial window of $8 \mathrm{~mm}$ was installed over the brain areas injected with YC3.6 as described above. After the surgeries, mice were allowed to recover from anesthesia on a heating pad. The mice were prepared for imaging 3-4 weeks after injection of virus. Mice received an intraperitoneal injection of methoxy-XO4 $(10 \mathrm{mg} / \mathrm{kg})$ and imaged with multiphoton microscopy through cranial windows the following day.
Chronic immunotherapy treatment. The mice in the chronic immunotherapy condition received intraperitoneal injections of ${ }^{\mathrm{ch}}$ aducanumab $(10 \mathrm{mg} / \mathrm{kg})$ or control antibody P1.17 $(10 \mathrm{mg} / \mathrm{kg})$ weekly for 6 months starting immediately after the baseline imaging session. Mice were 18 months of age at the start of treatment. The first two injections were made using Cy3-labeled antibodies to determine whether antibodies crossed the blood-brain barrier and colocalized with methoxy-positive plaques. Mice were imaged at baseline, 2 weeks after treatment initiation, and monthly in the course of the chronic immunotherapy treatment.

Multiphoton imaging and data acquisition. Imaging of YC3.6-filled neurons and amyloid plaques was performed using an Olympus Fluoview 1000MPE mounted on an Olympus BX61WI upright microscope (Kastanenka et al., 2015). A $25 \times$ water-immersion objective (numerical aperture 1.05) was used to visualize the brain and acquire images. A mode-locked titanium/sapphire laser (MaiTai; Spectra-Physics) generated two-photon fluorescence with 800 or $860 \mathrm{~nm}$ excitation. Detectors containing three photomultiplier tubes (Hamamatsu) collected light in the range of 380-480, 500-540, and 560-650 (Bacskai et al., 2001). Amyloid plaque pathology was imaged using $800 \mathrm{~nm}$ excitation at $1 \times$ magnification. A number or random $z$-stacks were taken within each cranial window at the first imaging session. The same regions within the cranial window were identified at later imaging sessions and reimaged. YC3.6-filled neurites and cell bodies were imaged with $860 \mathrm{~nm}$ excitation at $1 \times, 2 \times$, and $5 \times$ magnification. Laser power was maintained $<50 \mathrm{~mW}$ at the microscope back aperture to avoid phototoxicity. Image stacks were acquired at $1-5 \mu \mathrm{m}$ steps.

At the end of each imaging session, mice were allowed to recover on a heating pad. For subsequent imaging sessions, mice were injected with methoxy-XO4 the day before imaging, anesthetized, and imaged using the multiphoton microscope as described above. After the last imaging session, mice were killed with $\mathrm{CO}_{2}$, perfused with $4 \%$ paraformaldehyde in PBS and postfixed in $4 \%$ paraformaldehyde with $15 \%$ glycerol cryoprotectant for $24 \mathrm{~h}$. Brains were frozen in M1 mounting medium on ice-cold isopropanol, cut into $20 \mu \mathrm{m}$ coronal sections, and mounted onto slides. Amyloid plaques labeled with methoxy-XO4 were quantified. Four to 11 images per brain were acquired on a Zeiss Observer Z1 microscope with a $5 \times$ objective using MetaMorph software.

Image processing and data analysis. Image stacks acquired with multiphoton microscopy were analyzed using ImageJ. Images were processed and analyzed to elucidate amyloid plaque numbers and burden as well as baseline calcium. Because the same volumes of the brain before treatment and in the course of treatment were compared, each $z$-stack was verified to include the cells imaged in $z$-stacks acquired in prior imaging sessions for each brain volume. Cells not present in prior sessions were excluded from $z$-stacks analyzed. For amyloid plaque analysis, each $z$-stack was processed into a maximum intensity projection. Amyloid plaques were then manually counted in each projected image and compared before and after antibody treatment. For amyloid burden, each projected image was thresholded, segmented, and the percentage area occupied by amyloid was measured and represented as burden per cubic millimeter. Signal from amyloid lining the blood vessels was excluded from analyses. Amyloid plaque burden was calculated for cortical brain volumes imaged in vivo with multiphoton microscopy and cortical volumes and hippocampi imaged postmortem.

YC3.6 images were processed and analyzed using ImageJ. For both cyan fluorescent protein (CFP) and yellow fluorescent protein (YFP) channels, the background, corresponding to the mode at the last slice of each volume, was subtracted and a median filter with radius 2 was applied before dividing the emitted fluorescence intensity of YFP by CFP, thus creating a ratio image. Neurites and cell bodies were identified and selected using the YFP images either manually using the free hand tool or by adjusting the threshold and using the wand tool of the software. Selected regions of interest were then placed on the ratio images. Relative changes in the YFP/CFP ratio $\left(\Delta R / R_{\mathrm{i}}\right)$ were evaluated by selecting the same neurites or soma before and after treatment. Mice in which cranial windows failed to remain clear and thus made finding and analyzing same neurites impossible were excluded from the study.

YFP/CFP ratios were converted to $\left[\mathrm{Ca}^{2+}\right]_{\mathrm{i}}$ with standard equations using the in situ $K_{\mathrm{d}}$ and Hill coefficient of YC3.6 that we determined 
previously (Kuchibhotla et al., 2008). Pseudocolored images were created in MATLAB based on the YFP/CFP ratio, which was then converted to calcium concentration using the empirical $R_{\min }$ and $R_{\max }$ and assigned to the jet colormap. The ratio values were used to supply the hue and saturation (color) and the brightness values were used to supply the value (intensity).

Immunohistochemistry. Mouse brains were processed as described above. Cross-sections underwent antigen retrieval treatment in citrate buffer and were incubated with antibodies against NR1 (mouse, $100 \mu \mathrm{g} / \mathrm{ml}$, Millipore; RRID:AB_390129), NR2A (rabbit, $40 \mu \mathrm{g} / \mathrm{ml}$ Millipore; RRID:AB_310837), NR2B (rabbit, $20 \mu \mathrm{g} / \mathrm{ml}$ Millipore; RRID:AB_90772), Ryr (rabbit, $4 \mu \mathrm{g} / \mathrm{ml}$ Abcam; AB_1566701), SERCA2 (rabbit, $10 \mu \mathrm{g} / \mathrm{ml}$ Abcam), VILIP-1 (rabbit, $1.25 \mu \mathrm{g} / \mathrm{ml}$, Abnova; RRID:AB_1581891), IP3 receptor (rabbit, $1.3 \mu \mathrm{g} / \mathrm{ml}$, Abcam) for $2 \mathrm{~h}$ at room temperature followed by incubation with the appropriate secondary antibodies for $1 \mathrm{~h}$ and mounted with ProLong Antifade reagent (Invitrogen).

Statistics. Statistical analyses were performed in GraphPad version 5.0. Data are expressed as mean \pm SEM. Datasets were tested for normality (Shapiro-Wilk normality test, D’Agostino and Pearson omnibus normality test, or Kolmogorov-Smirnov test), after which the appropriate statistical tests were used (one-way $t$ test or ANOVA for normally distributed data, Mann-Whitney or Kruskal-Wallis test for nonparametric data). For datasets comparing 2 conditions, $p<0.05$ was considered significant. For datasets comparing 3 conditions, $p<0.025$ was considered significant.

\section{Results}

\section{Topical application of ${ }^{\mathrm{ch}}$ aducanumab clears plaques}

Amyloid plaque burden and amyloid plaque numbers were assessed after topical application of ch aducanumab directly to the surface of the brain. ${ }^{c h}$ aducanumab, a chimeric analog containing the intact variable domains of aducanumab, murine IgG2a heavy-chain, and murine $\kappa$ light-chain constant domains, was used instead of aducanumab itself because it mimics accurately the immune effector function in mice (e.g., the ability to bind Fc $\gamma$ receptors and recruit phagocytic cells). ${ }^{c h}$ aducanumab also reduces the likelihood of an antidrug response after repeat dosing in mice. ${ }^{\text {ch }}$ aducanumab $(0.4-1 \mathrm{mg} / \mathrm{ml})$ was applied directly onto the brain of living 22-month-old transgenic Tg2576 mice after craniotomy and durotomy. Multiphoton microscopy was used to image methoxy-XO4 labeled plaques on the day of the topical application and 3 weeks later to determine the effect of ${ }^{\mathrm{ch}}$ aducanumab on clearance of existing amyloid plaques and deposition of new plaques (Fig. 1A). We used landmarks in the mouse brains, such as cerebral amyloid angiopathy (CAA), to find the same brain regions and identify individual plaques reliably. Tg2576 mice used in this study had substantial CAA, the pattern of which was not altered with the antibody treatment. Acute topical application of ${ }^{\mathrm{ch}}$ aducanumab led to plaque clearance, determined as a decrease in the amyloid plaque number (Fig. $1 B-E, L$ ). A total of $48 \pm 4 \%$ of plaques disappeared after ${ }^{\text {ch }}$ aducanumab treatment compared with only $14 \pm 3 \%$ of plaques disappearing after treatment with a control antibody, P1.17 (Fig. 1J, MannWhitney test, $p<0.0001, n=31 z$-stacks in 3 mice treated with ch aducanumab, $n=39 z$-stacks in 4 mice treated with the control antibody). Furthermore, a decrease in size of $13 \pm 2 \%$ of the remaining individual plaques was observed after ${ }^{\mathrm{ch}}$ aducanumab treatment (Fig. 1M). Acute application of ${ }^{\mathrm{ch}}$ aducanumab did not prevent deposition of new plaques significantly (Fig. $1 B, D, K$; ch aducanumab: $34 \pm 5 \%$ of new plaques appeared vs control: $44 \pm 9 \%$; Mann-Whitney test, $p=0.98 ; n=31$-stacks in 3 mice treated with ${ }^{\mathrm{ch}}$ aducanumab, $n=39 z$-stacks in 4 mice treated with the control antibody). Overall amyloid plaque burden was reduced within the imaged volumes (Fig. $1 B-E, O$ ). Treatment with an isotype-matched monoclonal antibody with no known epitope in mouse brain, $\mathrm{P} 1.17$, did not result in amyloid plaque clearance (Fig. $1 F-J$ ), and there was a modest increase in amyloid burden in these mice over the 3 week period (Fig. 1O). Aducanumab treatment resulted in $27 \pm 8 \%$ reduction in amyloid plaque burden, whereas treatment with the control antibody resulted in a $33 \pm 15 \%$ increase in amyloid burden (Fig. $1 N$; MannWhitney test, $p<0.0001 ; n=31 z$-stacks in 3 mice treated with aducanumab, $n=39 z$-stacks in 4 mice treated with the control antibody). Therefore, a single topical application of ${ }^{\mathrm{ch}} \mathrm{adu}$ canumab led to a rapid decrease in amyloid burden as measured with longitudinal imaging.

\section{Chronic treatment with ${ }^{\text {ch }}$ aducanumab does not affect existing plaques in very old $\mathrm{Tg} 2576$ mice}

Brain penetration of the antibody was first determined using Cy3-tagged ${ }^{\mathrm{ch}}$ aducanumab injected intraperitoneally and colocalization with methoxy-XO4-positive plaques indicated target engagement in the brain (Fig. $2 A, B$ ). The control antibody also crossed the blood-brain barrier because $\mathrm{Cy} 3$ signal was visualized in mouse brains; however, it failed to colocalize with amyloid plaques (Fig. 2C,D). Six months of chronic treatment was initiated in 18-month-old animals that were imaged before antibody treatment (baseline), 2 weeks after onset of treatment, and once a month thereafter during the course of the treatment (Fig. $3 A$ ). Intraperitoneal injections of methoxy-XO4 were made before each imaging session. Because the same brain areas were imaged longitudinally in each mouse, amyloid plaque burden was determined and could be compared across imaging sessions. Amyloid plaque numbers were counted for the first and last imaging sessions. There was no substantial plaque clearance after the 6 month treatment period in either the chaducanumab- or the control-treated animals ( ${ }^{\mathrm{ch}}$ aducanumab: $4 \pm 1 \%$ of amyloid plaques were cleared; control: $5 \pm 2 \%$ of plaques cleared; MannWhitney test, $p=0.35 ; n=42 z$-stacks in 5 mice treated with chaducanumab, $n=22$-stacks in 3 mice treated with the control antibody), nor was there any evidence of substantial new plaque appearance ( ${ }^{\mathrm{ch}}$ aducanumab: $8.5 \pm 2 \%$ of new plaques appeared, control: $6 \pm 3 \%$ of new plaques appeared; Mann-Whitney test, $p=0.06 ; n=42 z$-stacks in 5 mice treated with ${ }^{\text {ch }}$ aducanumab, $n=22 z$-stacks in 3 mice treated with the control antibody) after control antibody (Fig. $3 E-K$ ) or ch aducanumab (Fig. $3 L-R$ ) treatment. Therefore, the net change in plaque number (Fig. $3 B$ ) or amyloid plaque burden (Fig. $3 C$ ) was not significant for either condition. The size of plaques was not altered as a result of ${ }^{\mathrm{ch}}$ aducanumab or control antibody treatment over the 6 month period ( $46 \pm 4 \%$ of amyloid plaques increased in size after ${ }^{\mathrm{ch}}$ aducanumab treatment vs $35 \pm 6 \%$ of plaques increased in size after control antibody treatment; Mann-Whitney test, $p=0.12$; $n=$ $42 z$-stacks in 5 mice treated with ${ }^{\text {ch }}$ aducanumab, $n=22 z$-stacks in 3 mice treated with the control antibody). Because in vivo multiphoton microscopy allows imaging of amyloid plaques in a small cortical volume, we confirmed the effects of immunotherapy using postmortem evaluation of amyloid burden in the cortex and hippocampus. After the final imaging session, brains were isolated, processed, and sectioned to allow for ex vivo quantification of methoxy-XO4-labeled amyloid plaques. Cortical amyloid plaque burden from in vivo imaging on the last imaging session was comparable to total cortical amyloid plaque burden analyzed postmortem and similar to that in hippocampus (Fig. 3D).

\section{Aged Tg2576 mice exhibit calcium overload}

Calcium homeostasis plays a major role in maintaining neuronal function. Resting calcium levels are elevated in aged APP mice, 
A
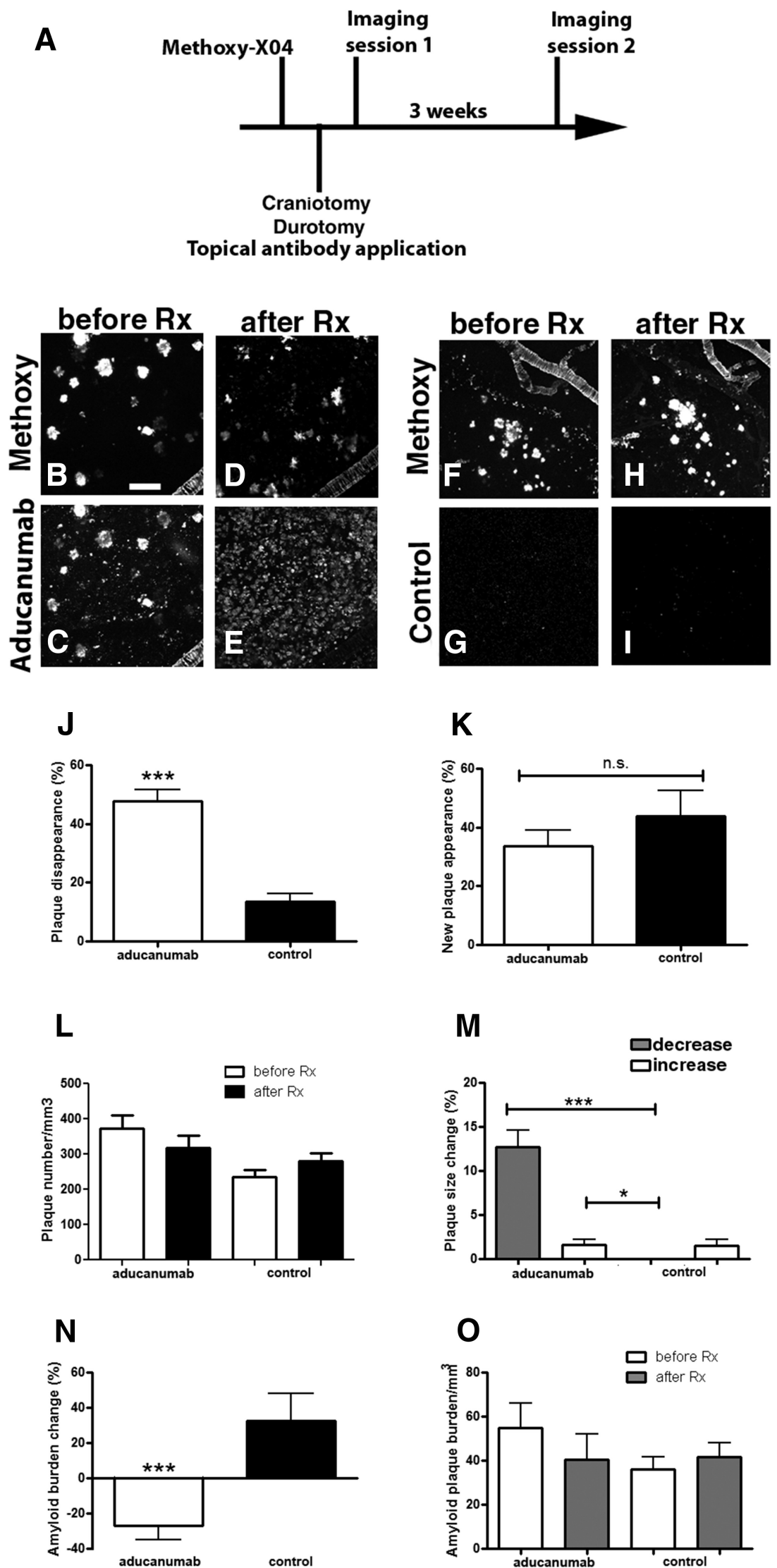

Figure 1. Topical application of chaducanumab leads to plaque clearance in old Tg2576 mice. A, Protocol for acute topical application of chaducanumab ( $n=3$ mice) or control antibody P1.17 ( $n=4$ mice) onto 22-month-old Tg2576 mouse brains followed by two-photon imaging. Methoxy-X04 was injected intraperitoneally before each imaging session. $\boldsymbol{B}-\boldsymbol{E}$, Two-photon images of methoxy-positive amyloid plaques $(\boldsymbol{B}, \boldsymbol{D})$ and cy3-labeled chaducanumab $(\boldsymbol{C}, \boldsymbol{E})$ in the cortex before the antibody resulting in calcium overload (Kuchibhotla et al., 2008). We tested whether chronic treatment with chaducanumab could restore neuronal calcium to control levels. Therefore, calcium dynamics were used as a measure of functional outcome.

First, we verified the presence of calcium overload in 18-month-old Tg2576 mice. Calcium was monitored using the ratiometric probe yellow cameleon 3.6 (YC3.6), which was virally expressed in the somatosensory cortex using an adenoassociated viral (AAV) vector before imaging (Fig. 3A). YC3.6 contains a donor, CFP, and an acceptor, YFP (Nagai et al., 2004). Binding of calcium to the probe leads to increased FRET efficiency and elevation in the ratio of YFP to CFP. Intracellular calcium concentrations can be derived directly from the ratio of YFP to CFP. Calcium overload was defined as a level $>2$ SDs above the mean of control cells. A ratio of 1.74 constituted calcium overload and translated into $200 \mathrm{nM}$ intracellular calcium concentration. Using multiphoton microscopy through cranial windows, we measured intracellular calcium concentration within neurites in 18-month-old transgenic Tg2576 mice when a substantial amyloid burden was present and in wild-type mice. A total of $18.5 \%$ of transgenic mouse neurites exhibited calcium overload, whereas only $4.0 \%$ of wild-type mouse neurites had elevated levels of calcium (Fig. $4 A, B$, black arrows) (Kuchibhotla et al., 2008). Therefore, aged Tg2576 mice exhibit elevated levels of baseline calcium within neurites.

Chronic treatment with ${ }^{\text {ch }}$ aducanumab restores intracellular calcium to control levels in aged $\mathrm{Tg} 2576$ mice

The effect of chronic treatment with chaducanumab on neuronal calcium overload was assessed in Tg2576 mice, which received systemic injections of $\mathrm{ch}_{\mathrm{adu}}$ canumab or control antibody at $10 \mathrm{mg} / \mathrm{kg}$

\footnotetext{
treatment (before $R x$ ) and after the treatment (after Rx). $\boldsymbol{F}-\boldsymbol{I}$, Twophoton images of methoxy-positive amyloid plaques $(\boldsymbol{F}, \boldsymbol{H})$ and cy3-labeled control antibody $(\mathbf{G}, \mathbf{I})$ before and after the antibody treatment.J, Percentage of plaques being cleared with antibodies. $\boldsymbol{K}$, Percentage of new plaques appearing across different conditions. $L$, Plaque number per cubic millimeter of cortical volume analyzed before and after treatment across conditions. $\boldsymbol{M}$, Percentage of individual plaques decreasing or increasing in size across conditions. $N$, Percentage of amyloid plaque burden changing as a result of treatment with antibodies (amyloid burden after treatment - amyloid burden before treatment). $\mathbf{0}$, Amyloid plaque burden before and after treatment across conditions. Scale bar, $100 \mu \mathrm{m}$. Data are shown as mean \pm SEM. ${ }^{*} p<0.05$, ${ }^{* * *} p<0.001$.
} 
A

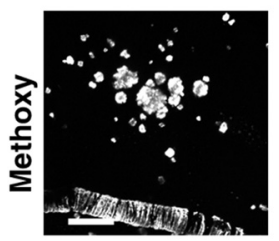

B

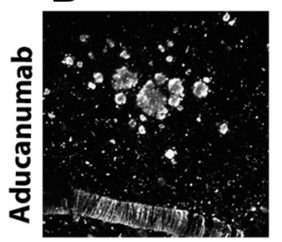

C

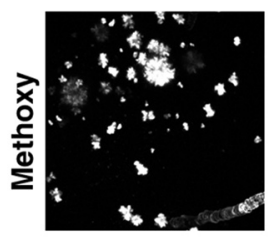

D

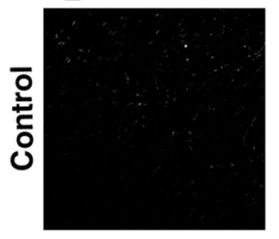

Figure 2. chaducanumab crosses the blood-brain barrier and colocalizes with methoxyX04-positive plaques. $\boldsymbol{A}-\boldsymbol{D}$, Colocalization of cy3-labeled chaducanumab $(\boldsymbol{B})$ with methoxypositive plaques in the cortex $(\boldsymbol{A})$. Lack of colocalization of cy3-labeled control antibody $(\boldsymbol{D})$ with methoxy positive plaques $(\boldsymbol{C})$ can be seen. Scale bar, $100 \mu \mathrm{m}$. weekly for 6 months starting at 18 months of age. YC3.6 was visualized before antibody treatment, 2 weeks after onset of treatment, and monthly thereafter in cortical neurites over the 6 month treatment period with multiphoton microscopy (Fig. 3A). Care was taken to image the same brain volumes during each imaging session and to select the same identified neurites during data analysis to make reliable comparisons with baseline calcium levels within each individual neurite over time. We used landmarks in the mouse brains such as neurite patterns, CAA, and amyloid plaques, the presence and location of which were comparable for a given brain across imaging sessions (Fig. $3 E-R$ ). Systemic administration of ${ }^{\mathrm{ch}}$ aducanumab restored neurite baseline calcium to control levels (Fig. 4C,D, yellow arrowheads). A total of $18.5 \%$ of neurites exhibited calcium overload at the start of treatment at 18 months (Fig. $4 A$, black arrow, $H$ ). Remarkably, chaducanumab decreased the number of neurites with elevated levels of calcium 2 weeks after the start of treatment ( 18.5 months vs 18 months in Fig. $4 A, H$ ). By the end of 6 months of treatment, at 24 months of age, mice treated with ${ }^{\mathrm{ch}}$ aducanumab had no

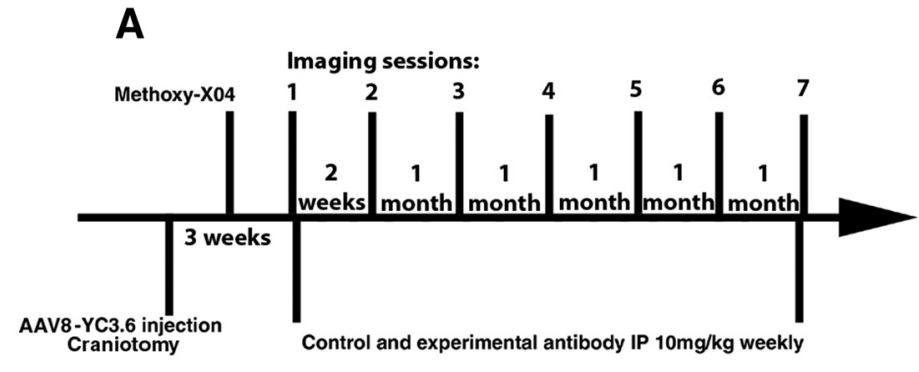

B
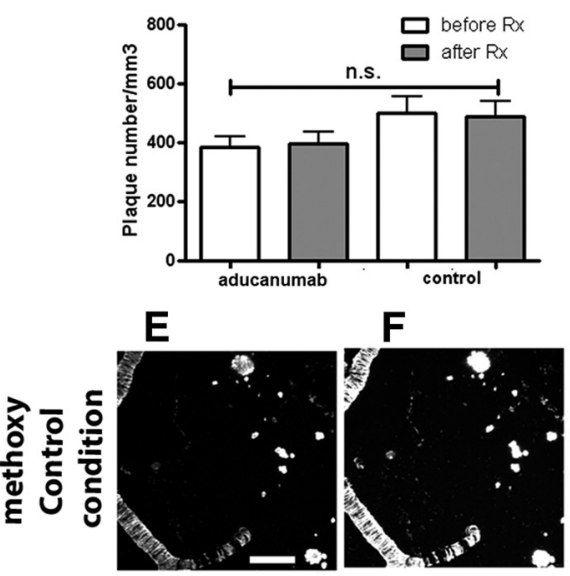

L

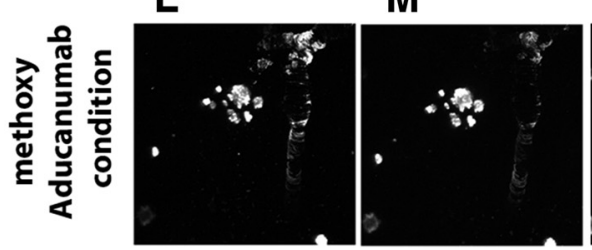

C

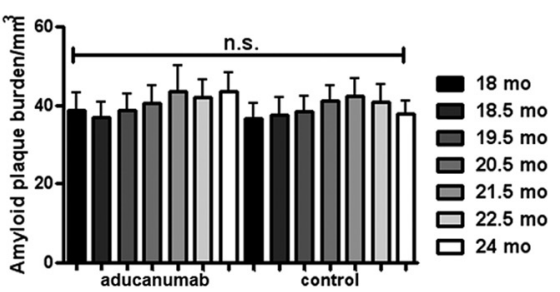

D

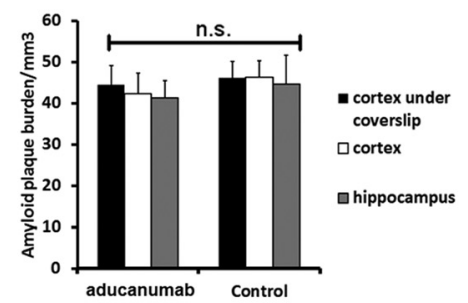

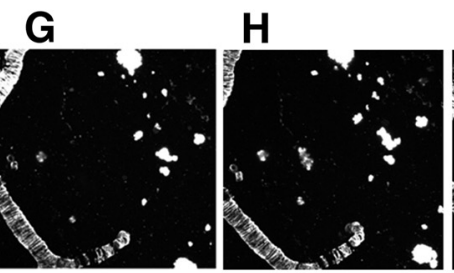
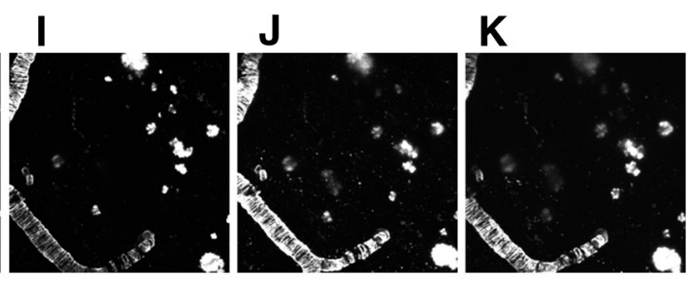

\section{$\mathrm{N}$}

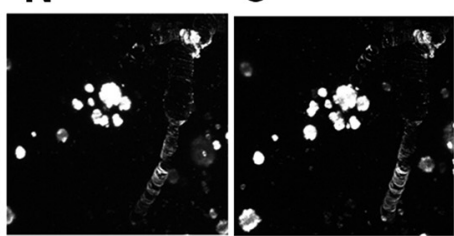

$\mathbf{P}$

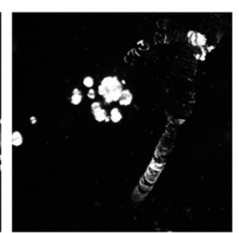

Q

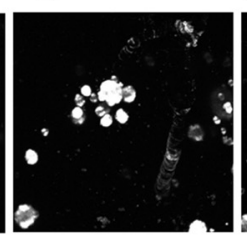

R

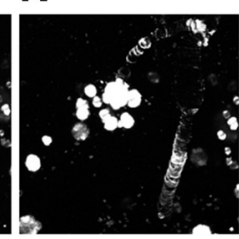

Figure 3. Systemic administration of chaducanumab does not clear existing plaques in very old $\mathrm{Tg} 2576$ mice. $A$, Protocol for chronic systemic injections of chaducanumab or control antibody into 18-month-old Tg2576 mice for 6 months and 2-photon imaging in the course of the treatment. Methoxy-X04 was injected intraperitoneally before each imaging session. $\boldsymbol{B}$, Plaque numbers before

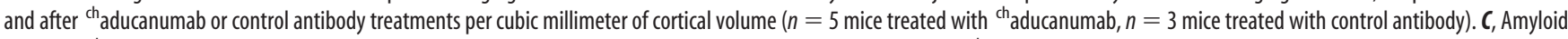
burden in chaducanumab-and control antibody-treated brains in the course of the treatment $\left(n=5\right.$ mice treated with ${ }^{\text {ch }}$ aducanumab, $n=3$ mice treated with control antibody). $\boldsymbol{D}$, Comparison of amyloid plaque burden imaged in the cortices via coverslips of cranial windows in vivo, postmortem cortices, and postmortem hippocampi. $\boldsymbol{E}-\boldsymbol{K}$, Two-photon images of amyloid plaques and CAA in the mouse cortex treated with control antibody. $\boldsymbol{L}-\boldsymbol{R}$, Two-photon images of amyloid plaques and CAA in the mouse cortex treated with chaducanumab antibody. Scale bar, $100 \mu \mathrm{m}$. Data are shown as mean \pm SEM. 
A

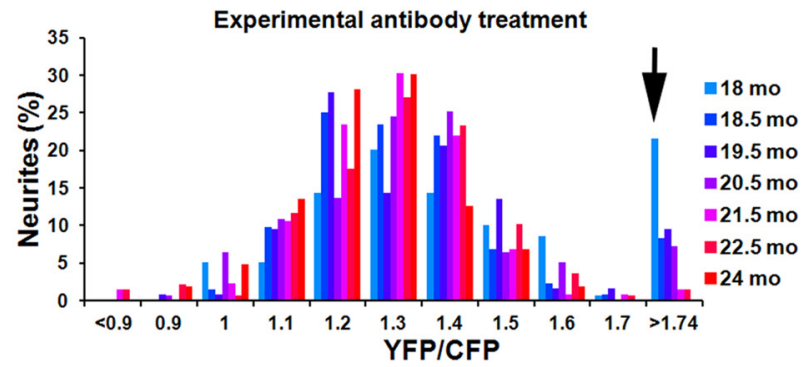

B
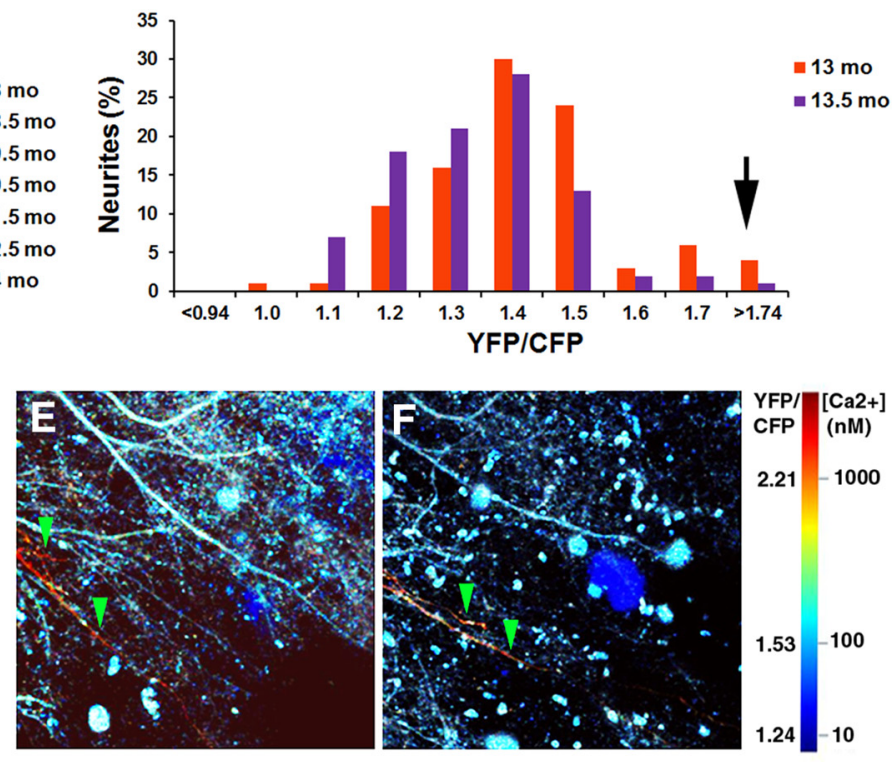
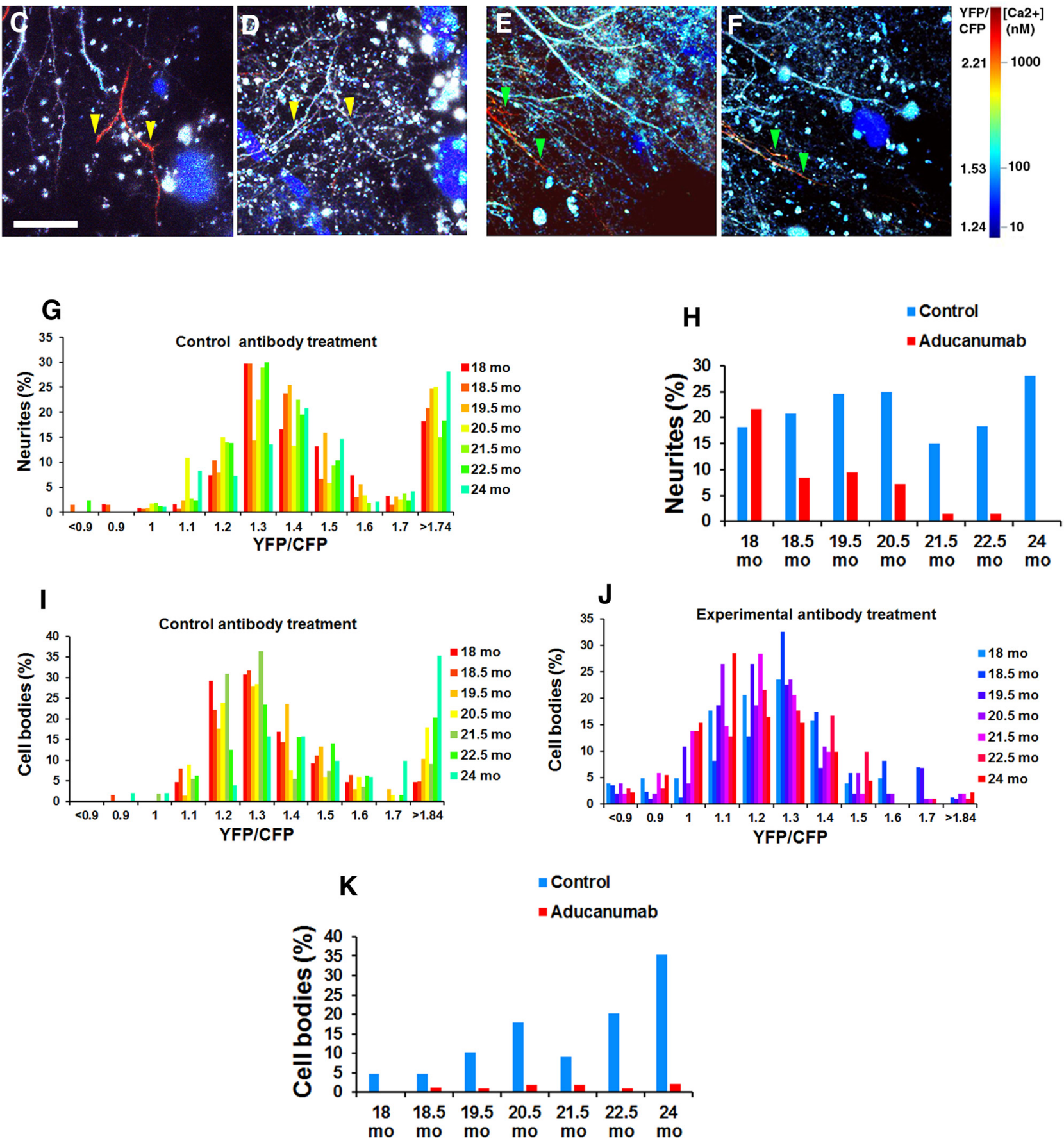

Figure 4. Neurite calcium overload is restored with chaducanumab immunotherapy treatment. $A$, Histograms of neurite YC 3.6 ratios for Tg2576 mice treated with ${ }^{\text {ch }}$ aducanumab throughout all

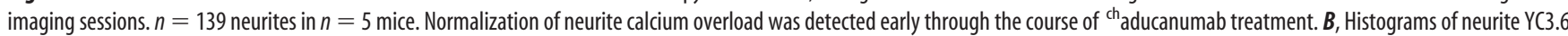
ratios for wild-type mice before and after two weekly treatments with chaducanumab. $n=96$ neurites in 3 mice. $\mathbf{C}-\boldsymbol{F}$, In vivo two-photon images of cortical neurites pseudocolored according to $\left[\mathrm{Ca}^{2+}\right]_{\mathrm{i}}$ show the presence of elevated levels of calcium (red neurites), whereas other neurites display normal calcium levels (blue neurites). (Figure legend continues.) 

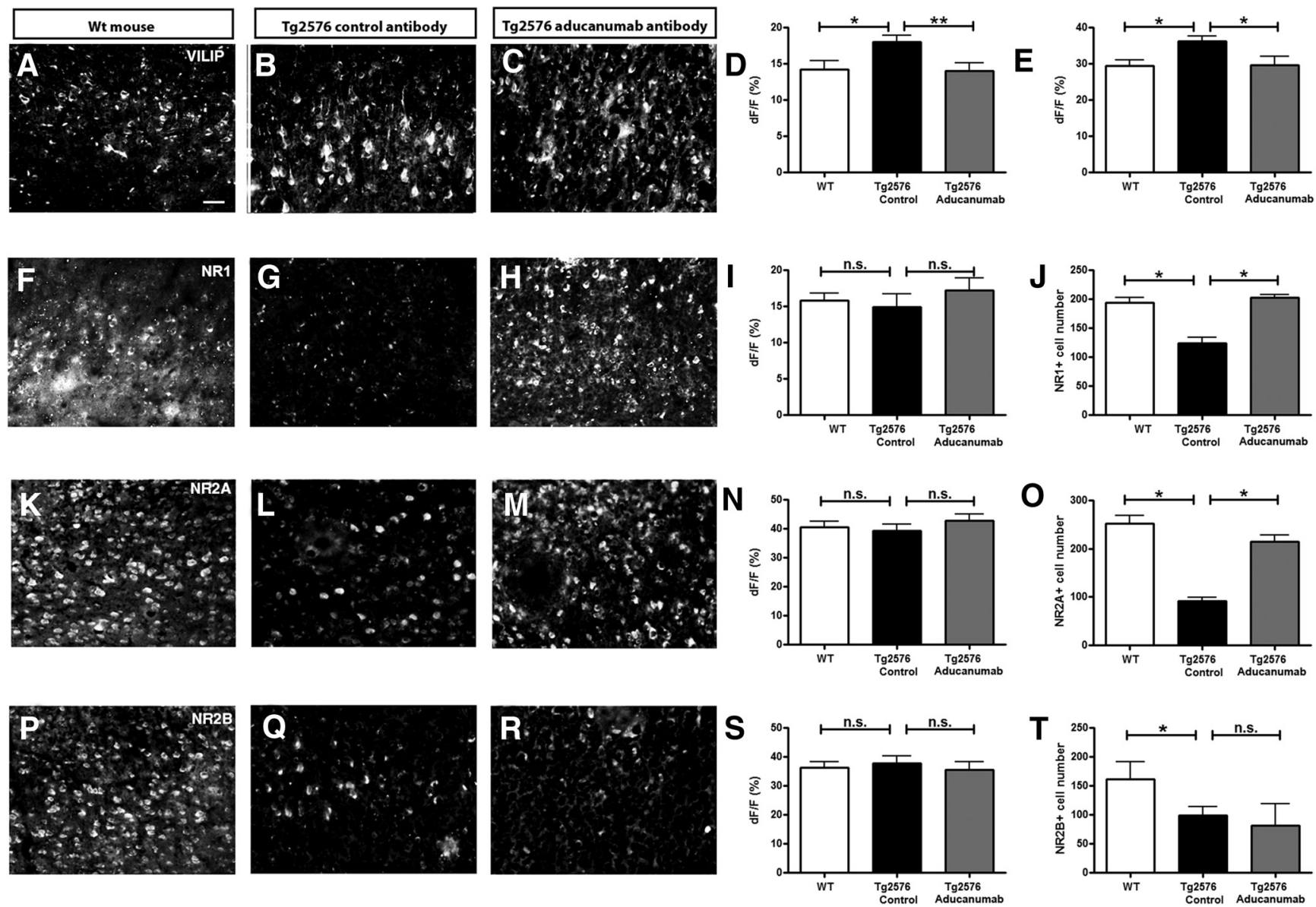

Figure 5. Immunohistochemical analysis of VILIP and NMDA receptor subunit expression in $20 \mu \mathrm{m}$ cortical sections of wild-type mice and Tg2576 mice chronically treated with control antibody or chaducanumab. Representative sections $(\boldsymbol{A}-\boldsymbol{C})$ and bar graphs summarizing fluorescence intensity of calcium sensor VILIP expression in cell bodies $(\boldsymbol{D})$ and neurites $(\boldsymbol{E})$ in the cortex $(n=3-4)$ are shown. $\boldsymbol{F}-\boldsymbol{J}$, NMDA subunit NR1 $(n=3-4)$, calcium-permeable NMDA subunits NR2A $(n=3-4)(\boldsymbol{K}-\boldsymbol{0})$, and NR2B $(n=3-4)(\boldsymbol{P}-\boldsymbol{T})$ protein expression in the cortex. $\boldsymbol{I}, \boldsymbol{N}, \mathbf{S}$, Fluorescence intensity measured within individual cells. $\boldsymbol{J}, \mathbf{0}, \boldsymbol{T}$, Number of cells positive for a given NMDA receptor subunit in the field of view. Scale bar, $50 \mu \mathrm{m}$. Data are shown as mean \pm SEM. ${ }^{*} p<0.05,{ }^{* *} p<0.01,{ }^{* * *} p<0.001$.

neurites with elevated levels of calcium (Fig. $4 \mathrm{~A}, \mathrm{H}$ ), whereas mice treated with the control antibody maintained elevated (28\%) calcium levels in neurites (Fig. 4E,F, green arrowheads, $G, H)$. Because individual neurites were identified and followed longitudinally, this reversal of calcium overload is not the result of neurite loss, but rather represents a true restoration of normal resting calcium. Furthermore, treatment with ${ }^{\mathrm{ch}}$ aducanumab did not alter baseline calcium in wild-type mice (Fig. $4 B$ ), suggesting that ${ }^{\text {ch }}$ aducanumab ameliorated calcium overload by interacting with human $\mathrm{A} \beta$ that $\mathrm{Tg} 2576$ mice produce due to overexpression

$\leftarrow$

(Figure legend continued.) chaducanumab restores neurite calcium to normal 6 months after initiation of treatment compared with 1 month after treatment initiation (cf. yellow arrowheads in $\boldsymbol{D}$ and $\boldsymbol{C}$ ). Treatment with control antibody does not restore calcium overload in neurites 5 months after initiation of treatment compared with 3 months after treatment initiation (cf. green arrowheads in $\boldsymbol{F}$ and $\boldsymbol{E}$ ). $\mathbf{G}$, Histograms of neurite $\mathrm{YC} 3.6$ ratios for $\mathrm{Tg} 2576$ mice treated with control antibody throughout all imaging sessions. $n=135$ neurites in $n=3$ mice in the control antibody condition. $\boldsymbol{H}$, Percentage of neurites exhibiting calcium overload in the course of the treatment. I, Histograms of cell body YC 3.6 ratios for Tg2576 mice treated with control antibody throughout all imaging sessions. $n=68$ neurites in $n=3$ mice in the control antibody condition. J, Histograms of neurites YC3.6 ratios for Tg2576 mice treated with chaducanumab throughout all imaging sessions. $n=102$ neurites in $n=4$ mice. $K$, Percentage of cell bodies exhibiting calcium overload in the course of the treatment. Scale bar, $100 \mu \mathrm{m}$. Data are shown as mean \pm SEM. of human APP and not by interacting with endogenous mouse $\mathrm{A} \beta$. Therefore, chaducanumab restored resting calcium to control levels and prevented neurite calcium overload in all neurites imaged ( ${ }^{\mathrm{ch}}$ aducanumab: $100 \%$ of neurites that started out with calcium overload had their calcium restored to control levels vs $4 \pm 3 \%$ of neurites in control antibody condition; Mann-Whitney test, $p<0.05 ; n=139$ neurites in 5 mice treated with chaducanumab, $n=135$ neurites in 3 mice in control antibody condition).

Furthermore, calcium levels were assessed in neuronal cell bodies. At the onset of treatment, Tg2576 mice exhibited limited calcium overload within their neuronal cell bodies $(5 \%$ in control

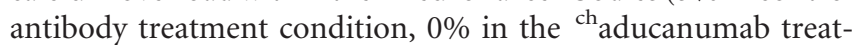
ment condition). The percentage of cell bodies exhibiting calcium overload increased in the course of treatment with the control antibody (Fig. $4 I, K)$. Interestingly, ${ }^{c^{2}}$ aducanumab treatment prevented this increase (Fig. $4 J, K$ ).

\section{NMDA receptors are downregulated in $\mathrm{Tg} 2576$ mice} exhibiting calcium overload

To gain insight into the cellular processes associated with neuronal calcium overload, we performed immunohistochemistry for a variety of calcium-homeostasis-related proteins in the mouse brains at the end of the chronic treatment period. The levels of the calcium sensor visinin-like protein (VILIP) (Tarawneh et al., 

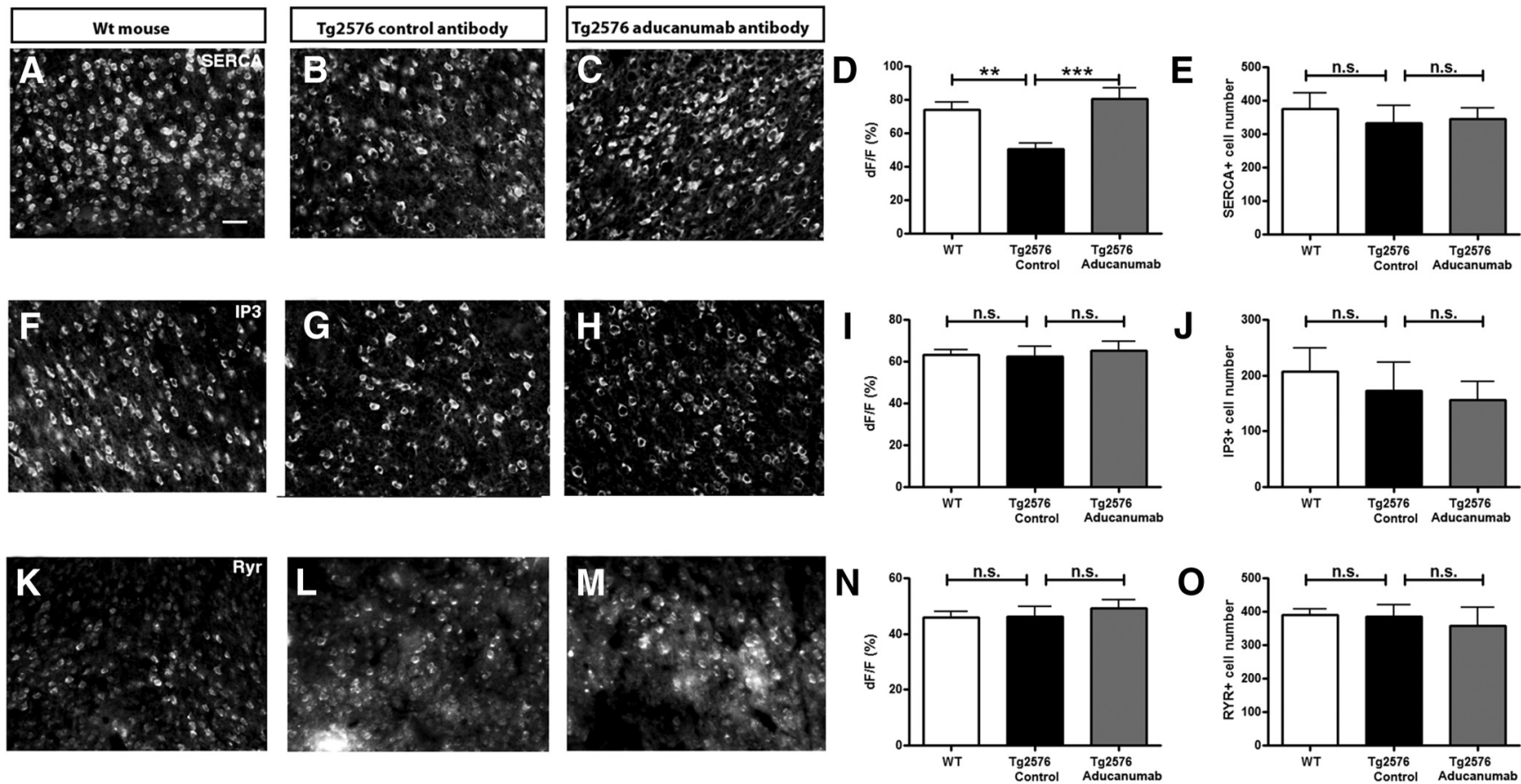

Figure 6. Immunohistochemical analysis of proteins associated with intracellular calcium store buffering system in $20 \mu \mathrm{m}$ cortical sections of wild-type mice and Tg2576 mice chronically treated with control antibody or chaducanumab. $\boldsymbol{A}-\boldsymbol{C}$, Representative sections and bar graphs summarizing SERCA fluorescence intensity (D) and cell body expression $(\boldsymbol{E})(n=3-4)$. $\boldsymbol{F}-\boldsymbol{J}, \mathrm{IP3}$ receptor expression and analysis $(n=3-4) . \boldsymbol{K}-\mathbf{0}$, RyR expression and analysis $(n=3-4)$. Scale bar, $50 \mu \mathrm{m}$. Data are shown as mean \pm SEM. ${ }^{*} p<0.05,{ }^{* *} p<0.01,{ }^{* * *} p<0.001$.

2011, Lu et al., 2015) were increased within neurites and cell bodies in Tg2576 mouse brains compared with the wild-type mice (Fig. $5 A, B, D, E$ ). Treatment with ${ }^{\text {ch }}$ aducanumab restored the levels of VILIP to control levels (Fig. $5 A, C, D, E$ ), corresponding to the restoration of calcium overload (Fig. $4 A, H$ ).

The NMDA receptor subunit NR1 and the NR2 subunits permeable to calcium, specifically NR2A and NR2B, were also assessed. The overall levels of these proteins were similar in the wild-type mice and the transgenic mice treated with control or

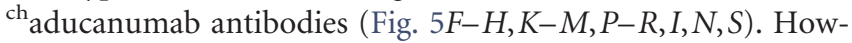
ever, when the numbers of cells within cortex were counted in the tissue sections positive for NR1, there were fewer NR1-positive neurons in Tg2576 mice treated with the control antibody compared with the wild-type controls (Fig. $5 F-H, J$ ). The number of NR2A- and NR2B-expressing cells also decreased in the mice treated with control antibody compared with wild-type mice (Fig. $5 K-M, O, P-R, T$ ). Treatment with ${ }^{c h}$ aducanumab restored the cell numbers to normal for NR1 and NR2A, but not NR2B (Fig. 5J,O,T).

Finally, the levels of proteins involved in regulation of internal calcium stores including calcium ATPase SERCA, IP3 receptor, and ryanodine receptor (RyR) (Rizzuto, 2001) were assessed. The number of SERCA positive cells did not differ significantly between conditions (Fig. 6A-C,E). However, levels of SERCA within the cells were reduced in Tg2576 mice treated with control antibody and restored to normal levels with ${ }^{\text {ch }}$ aducanumab treatment (Fig. 6A-D). Neither the cell number nor the levels of IP3 receptor and RyR was different between the two treatment groups or in wild-type mice (Fig. $6 \mathrm{~F}-\mathrm{O}$ ). These data suggest that the calcium overload is likely associated with aberrant entry of calcium from extracellular sources and that calcium homeostasis is restored by ${ }^{\text {ch }}$ aducanumab treatment.

\section{Discussion}

We evaluated the effects of aducanumab, an anti- $\mathrm{A} \beta$ antibody currently being evaluated in clinical trials for the treatment of $\mathrm{AD}$, on amyloid plaque clearance and restoration of intraneuronal calcium levels. Aducanumab is a human antibody that binds selectively to aggregated forms of $\mathrm{A} \beta$, including insoluble fibrils and soluble oligomers. We demonstrated that acute topical application of ${ }^{\mathrm{ch}}$ aducanumab, the murine analog of aducanumab, to the brain of Tg2576 mice resulted in clearance of existing amyloid plaques. Brain penetration and target engagement were demonstrated after systemic injection of the labeled antibody. Multiphoton microscopy was used for longitudinal imaging of very old Tg2576 mice treated with the antibody. Chronic systemic administration over 6 months with ${ }^{\text {ch }}$ aducanumab failed to clear existing amyloid plaques in these mice (18-24 months of age), consistent with the notion that immunotherapy is more effective for the prevention or treatment of amyloidosis at earlier stages and not as effective in advanced stages with substantial parenchymal plaque deposits. In addition, plaques in older animals might be resistant to anti-amyloid therapy, as was indicated in an earlier study (Demattos et al., 2012). However, despite the lack of detectable plaque clearance, a robust effect on intraneuronal calcium levels was observed. Future studies will be necessary to elucidate the effect of ${ }^{c h}$ aducanumab on calcium dynamics in astrocytes. Neuronal calcium dysregulation may be a mediator of progressive neurotoxicity (Kuchibhotla et al., 2008) and sustained elevations lead to activation of downstream effectors including the calcium-sensitive phosphatase calcineurin, which is associated with neurite pruning, loss of synapses, and neurotoxicity (Wu et al., 2010). Although no overt neurite pruning or cell body loss was observed in this study, a rigorous assessment will be necessary in future studies. Altered calcium homeostasis also leads to a shift in the balance of synaptic plas- 
ticity from an LTP-like state to one more resembling LTD (Berridge, 2011). Altogether, the dysregulation of intracellular calcium levels is likely to lead to functional abnormalities in neuronal networks that possibly underlie memory deficits in humans. Therefore, it is notable that anti-amyloid immunotherapy with ${ }^{\mathrm{ch}}$ aducanumab in Tg2576-transgenic mice can reverse this pathophysiological phenomenon and suggests that cognitive improvements can occur in the absence of overt amyloid plaque clearance.

A plausible explanation for this apparent discrepancy could be related to the specificity of ${ }^{\mathrm{ch}}$ aducanumab for aggregated forms of $\mathrm{A} \beta$. It has been demonstrated that $\mathrm{A} \beta$ oligomers are mediators of neurotoxicity and are likely to be the $\mathrm{A} \beta$ species that initiates the cascade of neurodegeneration (Crimins et al., 2013, Lesné et al., 2013). Therefore, the ability to target this species, in addition to clearing amyloid plaques, has important implications for the development of therapeutics. ${ }^{\text {ch }}$ aducanumab recognizes amyloid aggregates that includes soluble oligomers, but not monomers, of $\mathrm{A} \beta$. Therefore, this conformation-dependent antibody is well poised to not only avoid the overwhelming concentration of monomeric $A \beta$ in both plasma and brain parenchyma, but also to target the most relevant neurotoxic species in the brain. One can hypothesize that, by capturing soluble $A \beta$ oligomers, ${ }^{c h}$ aducanumab would prevent their binding to neuronal receptors known to be involved in calcium regulation, therefore preventing the calcium overload previously described in the brain of APPtransgenic mice.

To address the mechanisms of calcium dyshomeostasis and reversal with ${ }^{\text {ch }}$ aducanumab, we used immunohistochemical analysis of calcium related proteins in the brain. There were elevated levels of VILIP in cortices of Tg2576 mice treated with control antibody compared with levels in cortices of mice treated with ${ }^{\text {ch }}$ aducanumab. VILIP-1 has been identified as a brain injury biomarker and its levels are upregulated in $\mathrm{AD}$ patients (Tarawneh et al., 2011, Lu et al., 2015). Furthermore, whereas the NMDA receptor subunits NR1, NR2A, and NR2B were downregulated in APP mice, treatment with ${ }^{\text {ch }}$ aducanumab restored the levels of NR1 and NR2A, but not NR2B. It has been suggested that, in addition to being important as a developmental switch, the ratio of NR2A:NR2B also increases the susceptibility to excitotoxicity through the NMDA receptor (Tang et al., 1999, Cui et al., 2013). The expression of the intracellular store channel SERCA was reduced in Tg2576 mice treated with control antibody and restored with ${ }^{\mathrm{ch}}$ aducanumab immunotherapy, suggesting that intracellular calcium stores may contribute to calcium dyshomeostasis. However, RyR, calcium ATPase, and IP3 receptor levels were not different in transgenic compared with wildtype mice, suggesting that intracellular stores likely play a minor role.

In conclusion, treatment with ${ }^{\text {ch }}$ aducanumab led to the improvement of a functional outcome measure without reducing the amyloid load significantly after chronic treatment in very old mice. Therefore, we believe that an assay reflective of neuronal physiology, such as calcium imaging, might be considered as an indirect functional outcome measure. Assessment of cognitive deficits with behavioral tests in mice is conceptually appealing because this represents a direct functional end point. However, studies with anti-A $\beta$ immunotherapy have led to conflicting results, with effects on parenchymal $\mathrm{A} \beta$ often accompanied by no or very minor reversal of memory deficits (Arendash et al., 2001, Austin et al., 2003, Adolfsson et al., 2012, Bohrmann et al., 2012). Because ${ }^{\mathrm{ch}}$ aducanumab restored calcium overload, this antibody may have beneficial effects on neuronal network function that likely underlie cognitive deficits and is thus a promising treatment for AD. The development of conformationally specific immunotherapy approaches offers the opportunity to target the relevant amyloid species that initiate the neurodegenerative cascade without the confounding factor of engaging irrelevant $\mathrm{A} \beta$ species that limits overall efficacy.

\section{References}

Adolfsson O, et al. (2012) An effector-reduced anti-beta-amyloid (Abeta) antibody with unique abeta binding properties promotes neuroprotection and glial engulfment of Abeta. J Neurosci 32:9677-9689. CrossRef Medline

Arendash GW, Gordon MN, Diamond DM, Austin LA, Hatcher JM, Jantzen P, DiCarlo G, Wilcock D, Morgan D (2001) Behavioral assessment of Alzheimer's transgenic mice following long-term Abeta vaccination: task specificity and correlations between Abeta deposition and spatial memory. DNA Cell Biol 20:737-744. CrossRef Medline

Austin L, Arendash GW, Gordon MN, Diamond DM, DiCarlo G, Dickey C, Ugen K, Morgan D (2003) Short-term beta-amyloid vaccinations do not improve cognitive performance in cognitively impaired APP + PS1 mice. Behav Neurosci 117:478-484. CrossRef Medline

Bacskai BJ, Kajdasz ST, Christie RH, Carter C, Games D, Seubert P, Schenk D, Hyman BT (2001) Imaging of amyloid-beta deposits in brains of living mice permits direct observation of clearance of plaques with immunotherapy. Nat Med 7:369-372. CrossRef Medline

Bard F, et al. (2000) Peripherally administered antibodies against amyloid beta-peptide enter the central nervous system and reduce pathology in a mouse model of Alzheimer disease. Nat Med 6:916-919. CrossRef Medline

Bard F, et al. (2003) Epitope and isotype specificities of antibodies to betaamyloid peptide for protection against Alzheimer's disease-like neuropathology. Proc Natl Acad Sci U S A 100:2023-2028. CrossRef Medline

Berridge MJ (2011) Calcium signalling and Alzheimer's disease. Neurochem Res 36:1149-1156. CrossRef Medline

Bohrmann B, Baumann K, Benz J, Gerber F, Huber W, Knoflach F, Messer J, Oroszlan K, Rauchenberger R, Richter WF, Rothe C, Urban M, Bardroff M, Winter M, Nordstedt C, Loetscher H (2012) Gantenerumab: a novel human anti-Abeta antibody demonstrates sustained cerebral amyloidbeta binding and elicits cell-mediated removal of human amyloid-beta. J Alzheimers Dis 28:49-69. CrossRef Medline

Crimins JL, Pooler A, Polydoro M, Luebke JI, Spires-Jones TL (2013) The intersection of amyloid beta and tau in glutamatergic synaptic dysfunction and collapse in Alzheimer's disease. Ageing Res Rev 12:757-763. CrossRef Medline

Cui Z, Feng R, Jacobs S, Duan Y, Wang H, Cao X, Tsien JZ (2013) Increased NR2A:NR2B ratio compresses long-term depression range and constrains long-term memory. Sci Rep 3:1036. CrossRef Medline

Demattos RB, Lu J, Tang Y, Racke MM, Delong CA, Tzaferis JA, Hole JT, Forster BM, McDonnell PC, Liu F, Kinley RD, Jordan WH, Hutton ML (2012) A plaque-specific antibody clears existing beta-amyloid plaques in Alzheimer's disease mice. Neuron 76:908-920. CrossRef Medline

Dodart JC, Bales KR, Gannon KS, Greene SJ, DeMattos RB, Mathis C, DeLong CA, Wu S, Wu X, Holtzman DM, Paul SM (2002) Immunization reverses memory deficits without reducing brain Abeta burden in Alzheimer's disease model. Nat Neurosci 5:452-457. Medline

Doody RS, Thomas RG, Farlow M, Iwatsubo T, Vellas B, Joffe S, Kieburtz K, Raman R, Sun X, Aisen PS, Siemers E, Liu-Seifert H, Mohs R; Alzheimer's Disease Cooperative Study Steering Committee; Solanezumab Study Group (2014) Phase 3 trials of solanezumab for mild-to-moderate Alzheimer's disease. N Engl J Med 370:311-321. CrossRef Medline

Dunstan R, Bussiere T, Rhodes K, Engber T, Maier M, Weinreb P, Grimm J, Nitsch R, Arustu M, Qian F, Bussiere T, Li M (2011) Molecular characterization and preclinical efficacy. Alzheimers Dement 7:S457.

Kastanenka KV, Arbel-Ornath M, Hudry E, Galea E, Xie H, Bacskai BJ (2015) Optical probes for in-vivo imaging. In: Optical probes in biology (Zhang J, Schultz C, eds). New York: Taylor and Francis Group.

Klunk WE, Bacskai BJ, Mathis CA, Kajdasz ST, McLellan ME, Frosch MP, Debnath ML, Holt DP, Wang Y, Hyman BT (2002) Imaging Abeta plaques in living transgenic mice with multiphoton microscopy and methoxy-X04, a systemically administered Congo red derivative. J Neuropathol Exp Neurol 61:797-805. CrossRef Medline

Kuchibhotla KV, Goldman ST, Lattarulo CR, Wu HY, Hyman BT, Bacskai BJ 
(2008) Abeta plaques lead to aberrant regulation of calcium homeostasis in vivo resulting in structural and functional disruption of neuronal networks. Neuron 59:214-225. CrossRef Medline

Lesné SE, Sherman MA, Grant M, Kuskowski M, Schneider JA, Bennett DA, Ashe KH (2013) Brain amyloid-beta oligomers in ageing and Alzheimer's disease. Brain 136:1383-1398. CrossRef Medline

Lu H, Zhu XC, Jiang T, Yu JT, Tan L (2015) Body fluid biomarkers in Alzheimer's disease. Ann Transl Med 3:70. CrossRef Medline

Nagai T, Yamada S, Tominaga T, Ichikawa M, Miyawaki A (2004) Expanded dynamic range of fluorescent indicators for $\mathrm{Ca}(2+)$ by circularly permuted yellow fluorescent proteins. Proc Natl Acad Sci U S A 101: 10554-10559. CrossRef Medline

Rizzuto R (2001) Intracellular Ca2+ pools in neuronal signalling. Curr Opin Neurobiol 11:306-311. CrossRef Medline

Salloway S, et al. (2014) Two phase 3 trials of bapineuzumab in mild-tomoderate Alzheimer's disease. N Engl J Med 370:322-333. CrossRef Medline
Sevigny J, et al. (2016) The antibody aducanumab reduces Abeta plaques in Alzheimer's disease. Nature 537:50-56. CrossRef Medline

Tang YP, Shimizu E, Dube GR, Rampon C, Kerchner GA, Zhuo M, Liu G, Tsien JZ (1999) Genetic enhancement of learning and memory in mice. Nature 401:63-69. CrossRef Medline

Tarawneh R, D’Angelo G, Macy E, Xiong C, Carter D, Cairns NJ, Fagan AM, Head D, Mintun MA, Ladenson JH, Lee JM, Morris JC, Holtzman DM (2011) Visinin-like protein-1: diagnostic and prognostic biomarker in Alzheimer disease. Ann Neurol 70:274-285. CrossRef Medline

Wisniewski T, Goñi F (2014) Immunotherapy for Alzheimer's disease. Biochem Pharmacol 88:499-507. CrossRef Medline

Wu HY, Hudry E, Hashimoto T, Kuchibhotla K, Rozkalne A, Fan Z, Spires-Jones T, Xie H, Arbel-Ornath M, Grosskreutz CL, Bacskai BJ, Hyman BT (2010) Amyloid beta induces the morphological neurodegenerative triad of spine loss, dendritic simplification, and neuritic dystrophies through calcineurin activation. J Neurosci 30:2636-2649. CrossRef Medline 